\title{
CONSTRUCCIÓN DE IDENTIDAD Y ESTRUCTURAS DE PODER Y CONTROL EN EL AULA DE CLASE
}

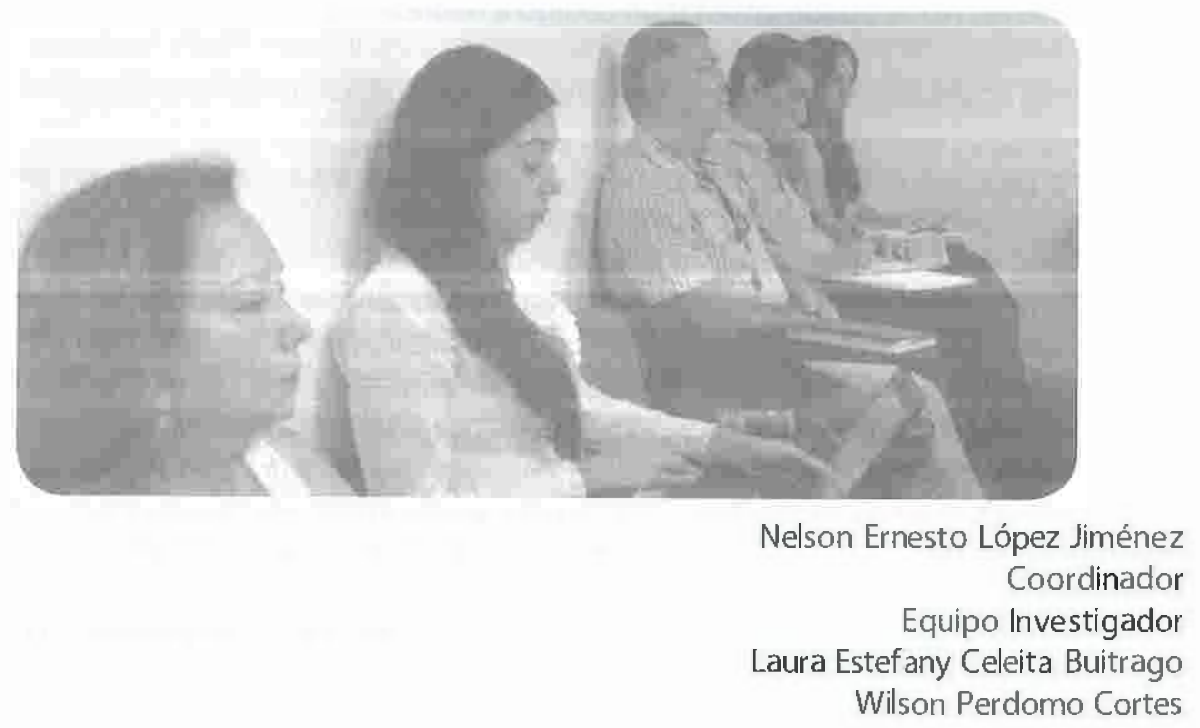

\section{RESUMEN:}

Dando continuidad a la línea de trabajo investigativo, el Grupo PACA, Categoría A de Colciencias, se presenta un estudio sistemático sobre las relaciones de poder y control que subyacen en el qula de clase y que a la post re contibu yen a $\mathrm{a}$ construcción de identidad profesional o subjetividad de los estudiantes.

Los conceptos de poder y control, en la teoría de Bernstein y continuadores (Días Villa, López Jiménez, Ortega, Cox, Daniels, Morais...) aunque están interrelacionados, son analíticamente diferentes y operan en niveles distintos. El poder crea límites, establece límites, legitima límites, reproduce límites entre categorías, sean estas agentes, agencias o discursos. E concepto de control, también establece límites pero ya no entre categorías, sino en el nivel de la comunicación. El control nos remite a la interacción, a las relaciones de poder de la interacciơn, a los límites de la interacción.

Se plantearon como objetivos principales el estudio de las estructuras de poderycontrol que permean y determinan la relaciones en el salón de clase $y_{c}$ a su vez, el impacto que tienen dichas relaciones en la construcción de subjetividades o identidades profesionales.

Dentro de la Teoría de la Transmisión Cultural (Bernstein y continuadores), se construyen dos conceptos fuertes en relación con el Poder y el Control, estos son la clasificación y la enmarcación. Se utiliza el concepto de clasificación para traducir las relaciones de poder y el concepto de enmarcación para traducir los principios de control social. Para estudiar la clas. 
ficación y la enmarcación se usó como dispositivo la categoría Código Educativo, entendido como "el conjunto de principios que regulan y estructuran un modelo pedagógico en sus dimensiones instruccional y regulativa. Es un principio regulador que se adquiere tácita e informalmente. Nose puede enseñar un código a nadie".

El problema analizado es la ausencia de estudios sobre los valores de los códigos y su influencia determinante en la construcción de identidad, que para el caso de la Universidad Surcolombiana, permitió determinar la identidad profesional de los egresados de sus diferentes programas, a partir del estudio de los códigos presentes.

日 diseño metodológico aplicado correspondió con un estudio descriptivo, analítico, explicativo y propositivo que permitió obtener como resultado, definir la identidad profesional de los egresados de b Universidad Surcolombiana, entendida esta como el resultado de la inser tar una carrera profesional en una base colectiva. La carrera profesional del estudiante es una carrera de conocimientos, una carrera moral y una carrera de situación. La base colectiva de esa carrera está constituida por el principio de orden social o et ordenamiento de lo social que se prevé transmita las universidades e institucionalice el Estado. (López: 2005).

E ar tículo pretende intervenir ytr ansformar el modelo pedagógico hegemónico vigente en la Universidad Surcolombiana.

\section{Planteamiento de b pregunta o problema de investigación y su justificación y refe- rente teórico:}

La Educación Superior, se constituye básicamente por agencias, agentes, discursos y prácticas que ocupan diferentes posiciones y entran en diferentes tipos de relaciones internas y externas. La universidad como objeto de estudio ha sido analizada desde diver sas perspectivas que dan cuenta de manera diferente sobre ella. Se afirma que la universidad conserva, memoriza, integra, ritualiza una herencia cultural de conocimientos, ideas, valores; la regenera examinándola, actualizándola, tr ansmitiéndola, también genera conocimientos, ideas y valores que se introducirán en la herencia. La universidad es conservadora, regeneradora, generadora (Morín: 1999). La universidad es pensada en têrminos de las dos funciones fundamentales que la definen como institución social y que constituyen su identidad: la producción de conocimiento, su distribución, socialización y confrontación con los problemas sentidos por las comunidades. ${ }^{12}$

En este contexto es válido recuperar lo planteado en la conferencia Mundial de la Educación Superior, realizada en París ${ }^{13}$ en julio de 2009 que hace alusión a lo siguiente:

12 En el lbro, El discurso pedagbglco oficial y la Edxación Superiar en Colombla, Olae y López, reallzan un análisis detallado sobre exe apecto. 2004.

13 Conferencla Mundial de Educadón Superior. UAESCO. Paris Julo de 2009 
- La Edaca ión Superior debe no solo dar herramientas sólidas para el mundo presente y futuro, sino contribuir a b educación de ciudadanos éticos comprometidos con la construcción de la paz, la defensa de los derechos humanos y los valores de la democracia.

- En los pasados 10 años se han hecho enormes esfuerzos para mejorar el acceso a la Educación Superior y asegurar la equidad. Estos esfuerzos continúan, sin embargo el simple acceso no basta, es necesario hacer mucho más; los esfuerzos deben asegurar el éxito en el aprendizaje.

- La Educación Superior, en su proceso de expansión del acceso, debe propender hacia la equidad, la relevancia y la calidad de forma simultänea. La equidad no es simplemente cuestión de acceso -el objetivo debe ser la participación y la culminación del proceso educativo, asegurando el bienestar de los estudiantes mediante el apoyo financiero y educativo a quienes forman parte de comunidades pobres y marginales.

- Las instituciones de educación superior a nivel mundial tiene la responsabilidad social de ayudar en el rompimiento de la brecha existente en términos de desarrollo entre los distintos paises, mediante el aumento de la transferencia de conocimientos más allá de las fronteras, especialmente hacia los países en vía de desarrollo. De igual manera deben trabajar en la búsqueda de soluciones comunes para fomentar la circulación de cerebros y aliviar los efectos negativos de la fuga de cerebros.

- Para una globalización de la Educación Superior en beneficio de todos, es vital asegurar la equidad en el acceso y el éxito de los procesos educativos; para ello, la educación debe promover el respeto a la diversidad cultural así como a la soberania nacional.

- Las Instituciones de Educación Superior deben buscar áreas de investigación y enseñanza que puedan apuntar hacia Cuestiones relativas al bienestar de $\mathbf{b}$ población y al establecimiento de una base local relevante y sólida en ciencias y tecnología.

Es importante señalar queen una sociedad, el auténtico desar rollo se estructura a partir de la formación de personas como su principal riqueza. Formación que garantiza una mirada holística de las diferentes dimensiones que constituyen la naturaleza humana.

Según la constitución Colombiana de 1991 b educación es un derecho fundamental, que debe ser esfuerzo permanente de los distintos estamentos para alcanzar un nivel que le permita desarrollarse como persona en escenarios de participación abierta. "Comprender y transformar a realidad, exige una interacción interdisciplinaria para lograr que los sistemas eduçativos cumplan con los objetivos sociales de ser universalmente incluyentes, para alcanzar una educación de calidad con desarrollo humano y colectivo, con eficacia y eficiencia "!"4

Se considera conveniente avanzar en l explicitación de una contextualización y conceptualización de la problemática investigada. Por ello se señala que el marco teórico está intimamente ligado con la Teoría de la Transmisión Cultural, entendida como "el conjunto de

14 Sarmiento Afredo. Lina estrategła para aumentar la retención de los estudiantes Mtyo 2006 
concepciones teóricas defendida y desarrollada por Basil Bernstein y continuadores que pretenden crear una Sociología de la Pedagogía. ${ }^{15}$

Las estructuras de poder y control que subyacen en la formación de los profesionales en la Educación Superior, se conceptualizan a partir de la metáfora del campo, es decir, "como resultado de relaciones de fuerza y de lucha para determinar las condiciones, los criterios de pertenecia y jerarquías legitimas, es decir, entre agentes y/o instituciones que tienden a conservar o a transformar formas especificas de poder en lo económico, político y cultural. Apropiando las ideas Bourdieu, Cristian Cox (1984) señala que la noción de campo, lleva intrínseca la noción de un espacio de posiciones (Dominante - dominado) estructurado en término de una disputa específica y generativo de competencias e intereses específicos".

La Teoría de la Transmisión Cultural da cuenta de la existencia de un campo pedagógico, entendido como el resultado de relaciones de fuerza y de lucha entre agentes y/o instituciones que tienden a conservar o transformar formas especificas de poder en lo económico, político y cultural; esto significa que no se comparte la idea o lectura que lo pedagógico se reduce a lo instrumental, instruccional o procedimental (bitácoras que hay que llenar o diseños y manuales que han de ejecutarse).

El concepto de práctica pedagógica nose circunscribe a las relaciones que tienen lugar en la escuela; la noción de práctica pedagógica se considera como un contexto social fundamental a través del cual se realiza la reproducción y la producción culturales, por ello, es gue entre las prácticas pedagógicas se incluyen las relaciones entre médico y paciente; las que existen entre el psiquiatra y el llamado enf ermo mental, las establecidas entre arquitectos y planificador es. ${ }^{16}$

La preocupación por develar las estructuras de poder y control gue subyacen en la formación de profesionales en la Universidad Surcolombiana necesariamente nos lleva a identificar la complejidad del campo en el cual sedan los procesos formativos, que para el caso de nos ocupa, será el aula de clase, en su sentido genérico y no como espacio físico.

Es necesario hacer un deslinde y señalar que bo formativo nose agota en lo escolar, si bien es un factor esencial, es necesario en tender queen $\mathbf{a}$ formación existen otras agencias culturales que también forman (la familia, el trabajo, la comunidad, el grupo social artístico, cultural, deportivo, etc), pero gue desafortunadamente no son analizadas de manera exhaustiva, cuando de estudios de abandono o ausentismo escolar se trata.

Bernstein produjo su teoría de los códigos para expresar los principios generales de estructuración y reproducción que articulan, en diferentes niveles analíticos, la división social del trabajo, la distribución de roles e identidades, la construcción de mensajes y significados, la distribución del poder y el ejercicio del control social (Díaz: 2000).

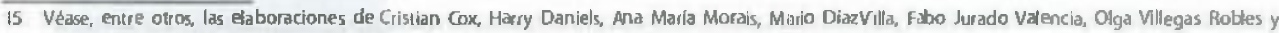

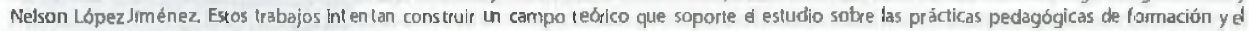
estudio de bs lenómenos inherentes a la realidad excoliar.

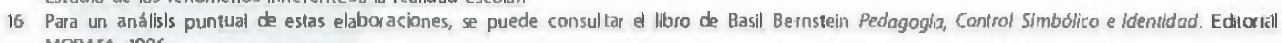
MORATA. 1996 
La naturaleza de los códigos difiere a partir de $\mathbf{a}$ dependencia de los contextos en los cuales se constituyen. Se afirma que cuando existe una dependencia marcada de las interacciones y procesos activados por los códigos de un contexto local, el código adquiere una naturaleza restringida y excluyente. Cuando la dependencia del código está referida a un contexto universal, adquiere una naturaleza elaborada, es decir, es mucha más amplia, robusta, incluyente y enriquecida. Uno de los elementos centrales que se constituye en la esencia del mejoramiento cualitativo de las IES hace referencia a la posibilidad de transformación sistemática y gradual de la naturaleza de los códigos educativos, es decir, de códigos restringidos avanzar a la consolidación de códigos elaborados. A partir de las anteriores enunciaciones teóricas se fundamenta el análisis de las Estructuras de Poder y Control (Clasificación y Enmarcación) presentes en la formación del profesional de la Universidad Surcolombiana.

El código educativo se entiende como "el conjunto de principios que regulan y estructuran un modelo pedagógico, en sus dimensiones instruccional y regulativa. Es un principio regulador y estructurador de prácticas pedagogicas. Los códigos y sus modalidades (ya sean elaborados o restringidos) son elementos intencionados que regulan la adquisición, reproducción y legitimación de reglas fundamentales de exclusión, inclusión y apropiación por y a traves de los cuales los sujetos son selectivamente creados, ubicados y controlados".

El poder opera siempre sobre las relaciones entre categorias, para Basil Bernstein, poder no se refiere a ninguna posesión, a ninguna propiedad, a ninguna cualidad o estado, sino a una categoria relacional. La metáfora básica para definir las relaciones de poder, es la metáfora agrícola de los límites, de los linderos; marcas que se crean en las relaciones internas y externas entre las categorías.

El control nos remite a la interacción, a las relaciones de poder de la interacción, a los límites de la inter acción. El control es portador de las relaciones de podery socializa a los individuos en esas relaciones. El control es un principio interactivo que permite legitimar o subvertir las relaciones de poder. El control establece formas legítimas de comunicacion apropiadas para las diferentes categorías. La relación maestro - estudiante, por ejemplo, es una relación de interacción, una relación cara a cara, a la cual subyacen principios de control ya través de los cuales se reproducen las relaciones de poder. El poder se relaciona con la división del trabajo y el control se relaciona con los principios de comunicación, con las formas de interacción. ${ }^{17}$

La investigación aquí proyectada, se fundamenta en los anteriores postulados conceptuales y persigue la elaboración de nuevo conocimiento, que se considera útil para el futuro deseable de la Universidad Surcolombiana, toda vez que pretende develar si se avanza en un proceso de reproducción o, por el contrario, se están creando nuevos espacios y nuevas formas de poder y control en los procesos académicos, investigativos, culturales, sociales, otros, inmersos en la formación de un profesional.

Los diferentes desarrollos investigativos que caracterizan a producción del Grupo de Investigación Paca, Categorfa A de Colciencias, advierten la necesidad de entender que el marco teórico de toda investigación, se debe leer como un resultado inherente a la investigación misma, razón por la cual, lo aquí planteado es una enunciación genérica del marco de referencia conceptual que orienta y determina la investigación pero que nose agota en esta enunciación.

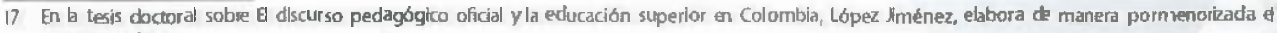
anterior análisis. 


\section{Naturaleza de la investigación:}

Se trabaja desde la perspectiva cualitativa y cuantitativa. Desde la perspectiva cualitativa a investigación propuesta se inscribe dentro de la corriente fenomenológica que privilegia la intencionalidad, la sub jetividad y las vivencias de los actores o protagonistas de la investigación, como fundamentos para la comprensión e interpretación del fenómeno a investigar.

\section{Resultados/Productos esperados y potenciales beneficiarios:}

- Conducentes al fortalecimiento de la capacidad científica nacional:

Este estudio investigativo aspira a convertirse en un referente válido y pertinente para el diseño de estrategias, dado que el impacto que se persigue, está relacionado con la intervención y transformación del modelo pedagógico hegemónico vigente en la Universidad Surcolombiana yen general de las Instituciones de Educación Superior del país.

En esa perspectiva los resultados obtenidos permiten avanzar en un proceso de análisis, reflexión sobre las estructuras de poder y control que subyacen en el proceso ce formación de profesionales en las diferentes áreas del conocimiento de la Universidad Surcolombiana. Dado el papel protagónico que ostenta el Grupo de Investigación PACA, categoría A de Colciencias, en la creación de la Red Nacional de Grupos de Investigación en el área de Educación en Colombia, los resultados encontrados aportaran elementos a b discusión investigativa a nivel nacional.

\section{Dirigidos a la apropiación social del conocimiento:}

Se espera que las Instituciones de Educación Superior del país conozcan, analicen y se apropien de manera concreta de los resultados de la investigación. Lo cual garantizará que ef estudio sobre las estructuras de poder y control que subyacen en el proceso de formación de profesionales en las diferentes áreas del conocimiento se convierta en referente válido y obligado para los procesos de enseñanza - aprendizaje gue permitan formar profesionales autónomos, creativos, agentes de transformación, cambio; comprometidos con la región y el pais.

\section{Bibliografía:}

Bernstein Basil: La Construcción Social del Discurso Pedagógico. Textos Seleccionados. Editorial PRODIC "El Griot". Segunda Edición, Bogotá, 1995.

Bernstein, Basil. Poder, educación y conciencia. Sociología de la transmisión cultural. Editor Cristian Cox CIDE. Santiago. 1988.

Bernstein, Basil. Hacia una Sociología de la Educación. Editores Mario Díaz, Nełson López. Colección Seminarium. Bogotá 2000. 
- La Estructura del Discurso Pedagógico. Clases, Códigos y Control.

Volumen N. Traducción Pablo Manzano. Editorial Morata. Segunda Edición. Madrid, 1994.

Pedagogía, Control Simbólico e Identidad. Teoría, Investigación y Crítica. Fundación Paideia, Ediciones Morata. Madrid. 1998.

. Conocimiento Oficial e Identidades Pedagógicas. Conferencia Pública en el Marco del Doctorado en Educación, Univalle, Cali. Mayo 7 de 1998.

Bernstein, B. y Diaz, M. Hacia una Teoría del Discurso Pedagógico. Revista Colombiana de Educación No.15 Primer Semestre de 1985. U.P.N. Bogotá.

CIFUENTES, Carlos, VANEGAS, Luz Ángela, APONTE, Claudia. Extensión Interactiva. Universidad de La Sabana. Chía, Colombia, Abril, 1997.

CONSEJO NACIONAL DE ACREDITACIÓN - CNA. Lineamientos para la acreditación institucional. Serie documentos 2.Bogotá, Colombia: 2005.

DE LA CRUZ AYUSO, Cristina y SASIÁ SANTOS, Pedro M. La Responsabilidad Social Universitaria en la Dimensión de la Extensión Universitaria. Aula de Ética de la Universidad de Deusto.Sf.

DIDRIKSSON, Axel. La nueva responsabilidad social y pertinencia de las universidades. Universidad Nacional Autónoma de México- UNAM. Ciudad de México, México. s.f.

HURTADO, Jackeline. "¿lnvestigación holística o comprensión holistica de la investigación?". En Revista Internacional Magisterio. No 31. febrero-marzo, 2008.

IAFRÁNCESCO Giovanni Modelo Pedagógico holístico Transformador. Fundamentos, dimensiones, programas y proyectos en la Escuela Transformadiora. CORIPET. Primera Edición. Bogotá. 2011

LÓPEZ Aragón, Wilson. Articulaciơn de la extensión con las otras funciones sustantivas y roles de los diferentes actores internos en la responsabilidad social universitaria. Universidad Santiago de Cali. Dirección general de Extensión. Cali, marzo de 2009.

LÓPEZ JIMÉNEZ, Nelson La De-construcción Curricular. Colección SEMINARIUM. Editorial MAGISTERIO, 2001.

LÓPEZ JIMÉNEZ Nelson, Ana Victoria Puentes y otros. Modelos y prácticas pedagógicas en la Educación Superior. Grupo PACA. Universidad Surcolombiana. Abril 2004.

- Modelos y prácticas pedagógicas en la Educación Superior. Grupo PACA. Univer sidad Surcolombiana. Abril 2004. 
. La Reestructuración Curricular de la Eđucación Superior. - Hacia la Integración del Saber-. ICFES - Universidad Surcolombiana. Bogotá, 1995.

LÓPEZ, JIMÉNEZ Nelson, Amaya Graciela y Otros. La Evaluación del Rendimiento Académico en los Programas a Distancia en Colombia. ICFES. Univer sidad del Quindio. Bogotá, 1990.

LÓPEZ, JIMÉNEZ Nelson, Mantilla, Magdalena y otros. Currículo y Calidad de la Educación Superior en Colombia. COLCIENCIAS - ICFES. Bogotá, 1989.

LÓPEZ, JMÉNEZ Nelson, Ramirez Jairo. El Tutor y el Desarrollo de la Capacidad Investigativa en la Educación Superior a Distancia. Proyecto Especial OEA-PREDE-8ó 117. Subdirección de Fomento. ICFES, Bogotá, Junio 1990.

LÓPEZ, JIMÉNEZ Nelson. El Proceso de Diseño, Desarrollo y Evaluación Curricular. 